\title{
A MAPA é Indicada para Todo Hipertenso e Normotenso? Existem Evidências para tanto?
}

\author{
Is ABPM Indicated for all Hypertensives and Normotensives? Is Supportive \\ Evidence Available?
}

Giovânio Vieira da Silva e Décio Mion Júnior

Universidade de São Paulo - São Paulo, SP

A hipertensão arterial é responsável diretamente por $80 \%$ dos casos de acidentes vasculares encefálicos e $40 \%$ dos casos de doença isquêmica do coração no Brasil, sendo essas duas condições responsáveis por $40 \%$ dos óbitos em nosso meio ${ }^{1}$. Apesar de não existirem estudos epidemiológicos de abrangência nacional, o Ministério da Saúde estima, baseado em levantamentos isolados, que a prevalência de hipertensão na população adulta brasileira é de pelo menos $20 \%{ }^{2}$. Portanto, o combate à hipertensão deve ser visto como uma prioridade em termos de Saúde Pública.

Dentro dessa visão, é mais do que óbvio dizer que tudo começa com um correto diagnóstico da hipertensão. Tradicionalmente, o diagnóstico da hipertensão é baseado em medidas de consultório, preferencialmente em duas ou mais ocasiões diferentes, de acordo com o preconizado nas IV Diretrizes Brasileiras de Hipertensão ${ }^{3}$. A informação prognóstica de que pessoas com valores de pressão arterial de consultório acima de 140/90mmHg estão sob maior risco cardiovascular é inquestionável em termos populacionais ${ }^{4}$. No entanto, algumas questões precisam ser levantadas.

Mesmo tomando-se todos os cuidados necessários para uma correta medida da pressão no consultório, que vão desde o correto posicionamento do paciente até o uso de esfigmomanômetros calibrados, dois aspectos inerentes a características biológicas da pressão arterial podem levar a medidas equivocadas: sua conhecida variabilidade em 24 horas, ou seja, uma medida isolada não representa necessariamente o comportamento da pressão durante todo o dia, e o efeito do avental branco, uma reação de alarme na presença do médico, que pode elevar significativamente a pressão arterial ${ }^{5}$.

\section{A MAPA e a medida de pressão no consultório}

A monitorização ambulatorial da pressão arterial (MAPA), pelas próprias características do método, além de sobrepujar tais limitações da pressão arterial de consultório, fornece uma série de outras informações sobre o comportamento da pressão arterial, tais como valores de média de pressão arterial em diferentes períodos do dia, pressão arterial durante o sono, carga pressórica e análises diversas sobre a variabilidade da pressão arterial (desviopadrão, early morning surge etc.) que, com maior ou menor importância, também se têm mostrado úteis em identificar pacientes com risco cardiovascular elevado $0^{6,7,8}$.

A superioridade da MAPA em relação à medida da pressão arterial de consultório não é apenas teórica. Em diversos estudos transversais, ao menos uma das variáveis obtidas na MAPA mostrou-se superior às medidas de consultório quando correlacionada com as mais diversas lesões em órgãos-alvo, seja hipertrofia miocárdica, infartos cerebrais lacunares, proteinúria, graus de retinopatia hipertensiva e espessura mio-intimal de carótidas ${ }^{9}$.

\section{A MAPA em hipertensos tratados e não tratados}

Vários estudos prospectivos tiveram como objetivo primário avaliar o papel da MAPA em prognosticar a ocorrência de eventos cardiovasculares em hipertensos não tratados, sendo que pelo menos duas variáveis da MAPA, a saber, as médias de pressão arterial e o descenso noturno da pressão arterial, demonstraram inequívocas vantagens da MAPA em identificar com mais propriedade aqueles pacientes que estavam sob maior risco $0^{10,11}$. À semelhança dos estudos prospectivos realizados em hipertensos não tratados, já dispomos de ao menos um estudo de coorte que analisou a MAPA em pacientes sob tratamento medicamentoso, sendo os resultados novamente favoráveis ao uso da MAPA ${ }^{12}$.

\section{A MAPA na população em geral}

Mais recentemente, dois estudos de coorte validaram esses resultados também para a população em geral. 0 estudo de Ohasama publicou dados referentes a 10 anos de seguimento de 1.332 indivíduos provenientes de uma comunidade japonesa do meio rural, dados que confirmaram observações já verificadas quando estas se limitaram aos cinco primeiros anos de acompanhamento: as médias de pressão arterial da MAPA (vigília, sono e 24 horas) são melhores marcadores de prognóstico cardiovascular do que a pressão de consultório,

Correspondência: Décio Mion Júnior - Av. Dr. Enéas de Carvalho Aguiar, 255 - 05403-000 - São Paulo, SP 
em particular a média de pressão arterial durante o sono ${ }^{13}$.

As críticas que poderiam existir acerca de extrapolação desses resultados para uma população ocidental e predominantemente urbana, como a brasileira, foram dissipadas com os resultados de um estudo dinamarquês que realizou a MAPA em 1.700 pessoas da população geral da cidade de Copenhague, que após um período médio de seguimento de 9,5 anos, também apontou informações prognósticas adicionais advindas da MAPA ${ }^{14}$. Assim como para a pressão de consultório, o estudo mostrou uma correlação positiva e linear entre a média de pressão arterial de 24 horas e o risco de mortalidade cardiovascular.

\section{Conclusões}

Portanto, apesar de a MAPA não estar presente no algoritmo diagnóstico de hipertensão de nenhuma sociedade internacional que tenha publicado uma diretriz relacionada ao tema, em um futuro não muito distante, dadas as vantagens da MAPA em relação à medida da pressão de consultório aqui colocadas, a indicação da MAPA para confirmar o diagnóstico de hipertensão poderá tornar-se obrigatória, sob pena de estarmos negando as melhores evidências disponíveis ${ }^{15}$.

Sem dúvida nenhuma, o grande empecilho para a imediata implantação dessa conduta é o custo proibitivo que tal abordagem traria para qualquer gestor de saúde que viesse a sustentá-la, seja ele público ou privado. No entanto, com o barateamento progressivo da tecnologia, o custo/efetividade desta abordagem ficará evidente.

A figura 1, adaptada de uma sugestão feita por Verdecchia ${ }^{16}$, exemplifica como a MAPA pode ser útil no diagnóstico de hipertensão, propiciando também uma estratificação adicional do risco cardiovascular, bem como sendo um guia na orientação terapêutica.

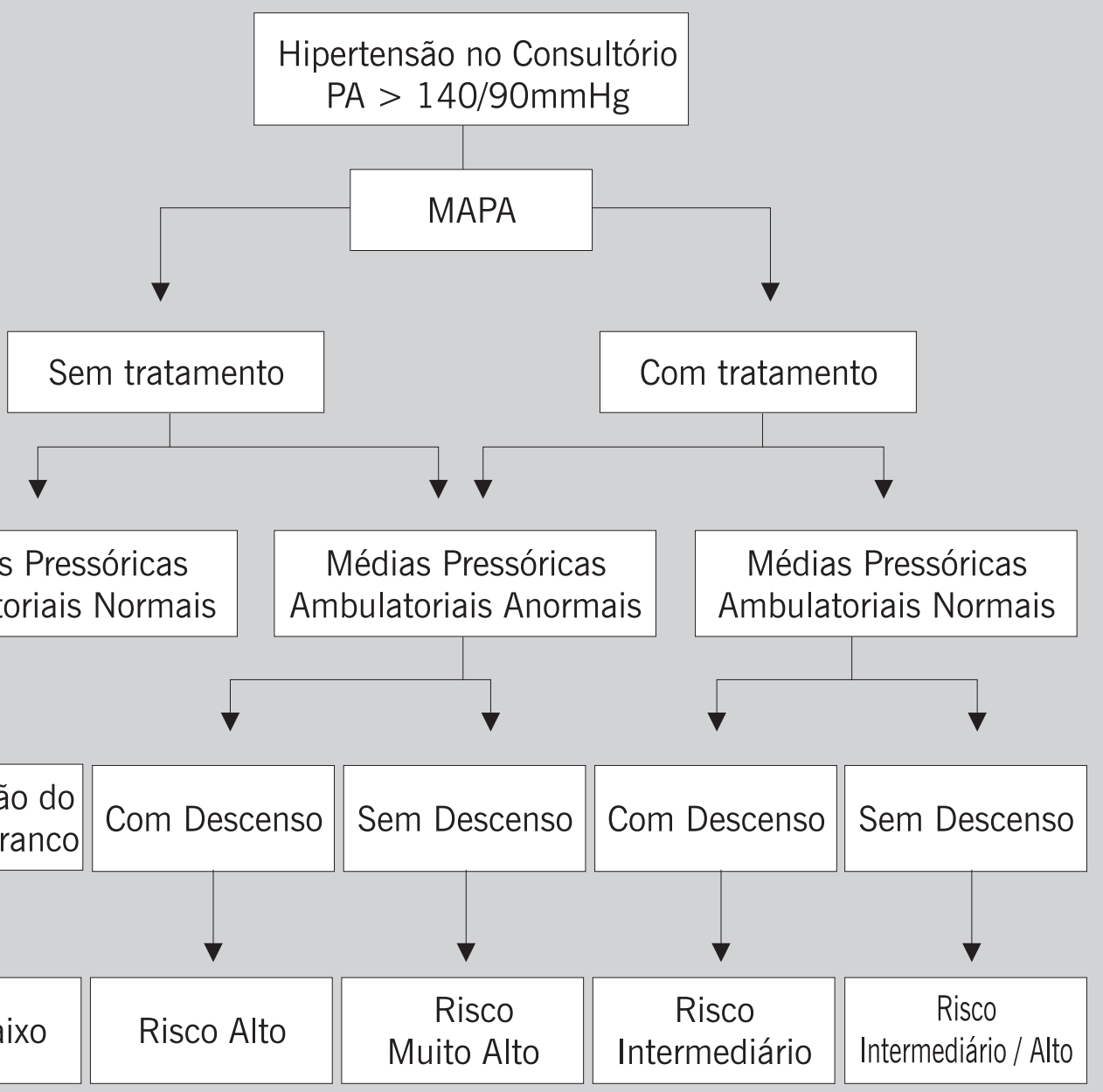

Fig. 1 - Estratificação do risco cardiovascular baseado nas médias pressóricas da MAPA e no descenso noturno da pressão arterial em pacientes tratados e não tratados. 


\section{REFERÊNCIAS}

1. Lotufo PA. Why Brazil does not have an outbreak of chronic diseases: lessons from cardiovascular diseases? Ciênc Saúde Coletiva 2004; 9 : 844-7

2. Guimaraes AC. Hypertension in Brazil. J Hum Hypertens 2002;16(Suppl 1):S7-S10

3. IV Brazilian guidelines in arterial hypertension. Arq Bras Cardiol 2004;82(Suppl 4):7-22.

4. Prospective Studies Collaboration. Age-specific relevance of usual blood pressure to vascular mortality: a meta-analisys of individual data for one million adults in 61 prospective studies. Lancet 2002;360:1903-13.

5. Pickering TG, DPhil, Hall JE et al. Recommendations for Blood Pressure Measurement in Humans and Experimental Animals Part 1: Blood Pressure Measurement in Humans A Statement for Professionals From the Subcommittee of Professional and Public Education of the American Heart Association Council on High Blood Pressure Research. Hypertension 2005;45:142-61.

6. Ohkubo T, Hozawa A, Yamaguchi J et al. Prognostic significance of the nocturnal decline in blood pressure in individuals with and without high 24-h bloodpressure: the Ohasama study. J Hypertens 2002;20:2183-89.

7. Kario K, Pickering TG, Umeda Y et al. Morning surge in blood pressure as a predictor of silent and clinical cerebrovascular disease in elderly hypertensives: a prospective study. Circulation 2003;107:1401-6.

8. Redon J, Liao Y, Lozano JV et al. Ambulatory blood pressure and microalbuminuria in essential hypertension: Role of circadian variability. J Hypertens 1994;12:947-53.
9. Verdecchia P, Angeli F, Gattobigio R. Clinical usefulness of ambulatory blood pressure monitoring. J Am Soc Nephrol 2004;15(Suppl 1):S30-3.

10. Perloff $D$, Sokolov M, Cowan R. The prognostic value of ambulatory blood pressure. JAMA 1983; 248: 2792-8.

11. Staessen J, Thijs L, Fagard R et al. For the Systolic Hypertension in Europe Trial Investigators. Prediccting cardiovascular risk using conventional vs ambulatory blood pressure in older patients with systolic hypertension. JAMA 1999;282:539-46.

12. Clement DL, Buyzere ML, Bacquer DA et al. For the Office vs Ambulatory Pressure Study Investigators. Prognostic value of ambulatory blood-pressure recordings in patients with treated hypertension. N Engl J Med 2003;348: 2407-15

13. Kikuya M, Ohkubo T, Asayama K et al. Ambulatory blood pressure and 10-year risk of cardiovascular and noncardiovascular mortality: the Ohasama study. Hypertension 2005;45:240-5.

14. Hansen TW, Jeppesen J, Rasmussen S, Ibsen H, Torp-Pedersen C. Ambulatory blood pressure and mortality: a population-based study. Hypertension 2005;45:499-504.

15. Myers MG. Ambulatory blood pressure monitoring for routine clinical practice. Hypertension 2005;45:483-4.

16. Verdecchia P. Prognostic value of ambulatory blood pressure. Current evidence and clinical implications. Hypertension 2000;35:844-51. 